\title{
Analysis of a Single-Wavelength Optical Buffer
}

\author{
K. Laevens and H. Bruneel \\ SMACS Research Group \\ Ghent University \\ Ghent, Belgium \\ $\{k 1, h b\} @$ telin.rug.ac.be
}

\begin{abstract}
We present a detailed analysis of the loss performance in an optical buffer having access to a single outgoing channel. Such a system - consisting of a number of fiber delay lines - differs significantly from a conventional electronic buffer, in that only a discrete set of delays can be realized for contention resolution. This leads to an underutilization of the channel capacity, which reduces overall performance. Our analysis does not require any special assumptions about the burst- or packet-size distribution, which allows us to study the impact this distribution has on performance. For the important special case of fixed-sized bursts, it reveals, amongst others, that matching fiber delay line length with burst duration is not necessarily the optimal solution in terms of loss performance. It further reveals that, in general, this optimal solution is function not only of burst-size characteristics, but of the offered load as well, making the buffer design process a delicate task.
\end{abstract}

Keywords - burst switching; fiber delay lines; loss probability; optical buffers

\section{INTRODUCTION}

As advances in Dense Wavelength Division Multiplexing (DWDM) push fiber transmission capacities well beyond the Tbit/s, electronic switches and routers are becoming the bottlenecks of the backbone network. All-optical packet switching (OPS) could alleviate the problem, but the technology is expected to be still a few years away [1]. As intermediate solution, optical burst switching (OBS) has been proposed by some [2]-[4]. Both solutions, however, confront switch designers with several major challenges, of which the lack of optical RAM is but one. Fiber delay lines (FDLs) partially fill that gap, and several architectures based thereon have already been studied [5]-[7].

Few analytic results concerning the loss and queueing behavior of such buffer systems have been published so far [8], and to the best of the authors' knowledge the approach followed here is new. Our main result is the derivation of the probability generating function of the scheduling horizon in a buffer of infinite size, as seen by arrivals. This scheduling horizon can be thought of as being the analogue of the unfinished work or virtual waiting time in classical queueing theory. From that result - the detailed derivation of which we choose to present as Appendix - several measures of interest are then derived, such as the system capacity and the loss probability in a finite system. Although the analysis can easily be extended to yield results concerning e.g. sojourn times, we primarily focus on loss probability here, given that this figure will be the determining factor for the system's overall performance.

\section{MODEL}

We focus on a single WDM channel and assume contention for it is resolved by means of an FDL buffer, which can delay, if necessary, optical bursts (or packets) until the channel becomes available again. Unlike conventional buffers, however, it can not delay bursts for an arbitrary period of time, but only for multiples of a basic unit $\mathrm{D}$, called the granularity of the FDLs [8]. Each burst is thus either delayed $\mathrm{n} \cdot \mathrm{D}$ time units, for some $\mathrm{n}=0,1, \ldots \mathrm{N}$, or is dropped. Hereby, $\mathrm{N}$ is the size of the FDL buffer, the maximum achievable delay being N.D.

We assume time is slotted and we will use the duration of a single slot as the unit of time. A natural choice for the slot length would be the clock cycle in a synchronous system. By making the length of a slot sufficiently small, however, and scaling everything accordingly, results for an asynchronous system can be retrieved as well.

Thus, for instance, the granularity D would be expressed as an integer number of slots. Note that when $\mathrm{D}$ equals one (slot), the optical buffer functions as a conventional timeslotted one, i.e., it is no longer degenerate, since then any delay in the range 0 to $\mathrm{N}$ can be realized.

The sizes of consecutive bursts will be denoted by $B_{k}$ $(\mathrm{k}=1,2, \ldots)$, expressed as number of slots needed for their transmission. We assume the $B_{k}$ form a sequence of iid (independent and identically distributed) random variables (rv's), distributed according to some common distribution $\operatorname{Pr}\left[\mathrm{B}_{\mathrm{k}}=\mathrm{n}\right]$, with mean $\mathrm{E}[\mathrm{B}]$ and probability generating function (pgf)

$$
\mathrm{B}(\mathrm{z})=\mathrm{E}\left[\mathrm{z}^{\mathrm{B}_{\mathrm{k}}}\right]=\sum_{\mathrm{n}=1}^{\infty} \mathrm{z}^{\mathrm{n}} \operatorname{Pr}\left[\mathrm{B}_{\mathrm{k}}=\mathrm{n}\right]
$$

Note that no further specific assumptions are needed in the remainder of the analysis.

The first author would like to acknowledge the Flemish Government for support of his research through the IWT-GBOU project "Optical Networking and Node Architectures". 


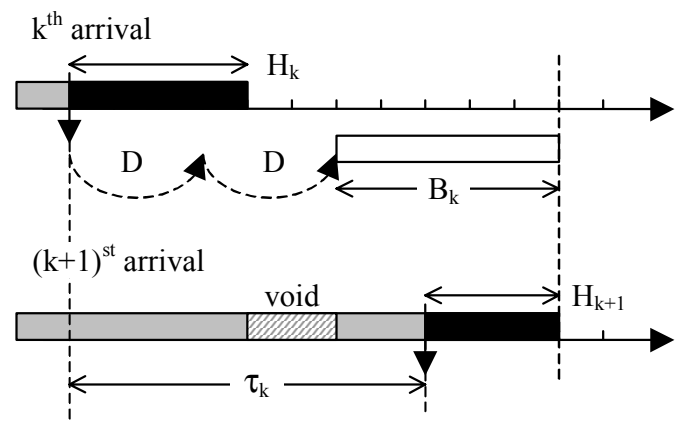

Fig. 1. Evolution of the scheduling horizon $\mathrm{H}$ from one arrival to the next. For notation, please refer to the text.

As for the arrival process, we assume that at most one new burst can arrive during each slot, which happens with probability $\mathrm{p}$ independently from slot to slot. That is, we assume Bernoulli arrivals, the counterpart of the Poisson process in continuous-time models. Burst interarrival times, denoted $\tau_{\mathrm{k}}$, are then iid rv's as well, with mean $1 / \mathrm{p}$ and a geometric distribution

$$
\operatorname{Pr}\left[\tau_{\mathrm{k}}=\mathrm{n}\right]=\mathrm{p} \cdot \overline{\mathrm{p}}^{\mathrm{n}-1} \quad(\mathrm{n}=1, \ldots) .
$$

(We hereby adopted the standard convention of denoting 1-p by $\overline{\mathrm{p}}$.) This geometric distribution can be shown to be memoryless, in accordance with the Bernoulli arrival process.

\section{ANALYSIS}

\section{A. Main result}

Let us assume the buffer is of infinite size, so that no loss occurs. Denote by $\mathrm{H}_{\mathrm{k}}$ the scheduling horizon as seen by the $\mathrm{k}$ th arrival. It is, by definition, the earliest time (measured in slots) by which all previously arrived bursts will have left the system, and is thus, as mentioned before, the equivalent of the virtual waiting time in conventional queues.

In Fig. 1, the relation between $\mathrm{H}_{\mathrm{k}}$ and $\mathrm{H}_{\mathrm{k}+1}$ is illustrated. With that figure at hand, it is rather straightforward to establish that

$$
\mathrm{H}_{\mathrm{k}+1}=\left[\mathrm{B}_{\mathrm{k}}+\mathrm{D} \cdot\left\lceil\frac{\mathrm{H}_{\mathrm{k}}}{\mathrm{D}}\right\rceil-\tau_{\mathrm{k}}\right]^{+}
$$

Hereby, $\lceil\mathrm{x}\rceil$ is the so-called ceil of $\mathrm{x}$, the smallest integer greater than or equal to $\mathrm{x}$. The standard notation $[\mathrm{x}]^{+}$is shorthand for $\max (0, \mathrm{x})$.

When the k-th burst sees a scheduling horizon $\mathrm{H}_{\mathrm{k}}$ upon arrival, it will have to be delayed by at least that amount to avoid contention. Since the buffer is degenerate, however, this delay can not be realized exactly, the closest match being given by $D \cdot\left\lceil\mathrm{H}_{k} / \mathrm{D}\right\rceil$. (Note that $\left\lceil\mathrm{H}_{k} / \mathrm{D}\right\rceil$ represents the index of the FDL the k-th burst must traverse.) Delaying and transmitting this burst pushes the scheduling horizon (just after arrival) to

$$
\mathrm{B}_{\mathrm{k}}+\mathrm{D} \cdot\left\lceil\frac{\mathrm{H}_{\mathrm{k}}}{\mathrm{D}}\right\rceil
$$

Taking then into account the burst interarrival time $\tau_{\mathrm{k}}$ immediately gives (3).

When the system is stable, i.e., when it can reach stochastic equilibrium, the distributions of the $\mathrm{H}_{\mathrm{k}}$ converge, for $\mathrm{k} \rightarrow \infty$, to a unique equilibrium distribution, which does not depend on the initial condition of the system. Associated with this distribution is a common pgf, denoted $\mathrm{H}(\mathrm{z})$, whose exact form is derived in Appendix.

\section{B. System Capacity}

The finite granularity of the FDLs leads to voids, as illustrated in Fig. 1, reducing channel utilization and lowering the system's overall capacity.

A system with an infinite (optical) buffer does not reach steady-state when overloaded, but grows unbounded, reflected in e.g. the fact that

$$
\lim _{\mathrm{k} \rightarrow \infty} \operatorname{Pr}\left[\mathrm{H}_{\mathrm{k}}=0\right]=0 \text {. }
$$

Based on this, one can define a maximum tolerable arrival intensity $\mathrm{p}_{\max }$. From the results in Appendix, it follows that $\mathrm{p}_{\max }$ is solution of

$$
\mathrm{p}_{\max }=\left(\mathrm{E}[\mathrm{B}]+\frac{\mathrm{D}-1}{2}+\sum_{\mathrm{k}=1}^{\mathrm{D}-1} \frac{1}{\varepsilon_{\mathrm{k}}-1} \frac{\mathrm{p}_{\max } \mathrm{B}\left(\varepsilon_{\mathrm{k}}\right)}{\varepsilon_{\mathrm{k}}-\overline{\mathrm{p}}_{\max }}\right)^{-1}
$$

(The symbols $\varepsilon_{\mathrm{k}}$ represent the $\mathrm{D}$ different complex $\mathrm{D}^{\text {th }}$ roots of unity, see Appendix.) The solution is thus function of the FDL granularity D and the (complete) burst-size pgf $\mathrm{B}(\mathrm{z})$, or, equivalently, the (complete) burst-size distribution.

A sufficiently close approximation, function of $\mathrm{D}$ and the mean burst size $\mathrm{E}[\mathrm{B}]$ only, is given by

$$
\mathrm{p}_{\max } \approx\left(\mathrm{E}[\mathrm{B}]+\frac{\mathrm{D}-1}{2}\right)^{-1}
$$

consistent with the observation made in e.g. [8], that each burst creates a void on the channel of size, on average, about $\mathrm{D} / 2$.

With this maximum tolerable arrival intensity corresponds a maximum tolerable load, given by 


$$
\rho_{\max }=\mathrm{p}_{\max } \cdot \mathrm{E}[\mathrm{B}] \approx\left(1+\frac{\mathrm{D}-1}{2 \cdot \mathrm{E}[\mathrm{B}]}\right)^{-1},
$$

which is thus, in general, less than unity.

\section{Loss Probability}

Estimates for the loss probability (LP) in an (optical) buffer of finite size can be derived from the distribution of the unfinished work in a system of infinite size, which quantity we will denote by $\mathrm{H}_{\text {inf }}$ from now on. Given the maximum achievable delay in the finite system is N.D, one can use the heuristic

$$
L P \approx\left(1-\rho_{\mathrm{eq}}\right) \frac{\operatorname{Pr}\left[\mathrm{H}_{\mathrm{inf}}>\mathrm{N} \cdot \mathrm{D}\right]}{1-\operatorname{Pr}\left[\mathrm{H}_{\mathrm{inf}}>\mathrm{N} \cdot \mathrm{D}\right]},
$$

where $\rho_{\mathrm{eq}}$ is defined as

$$
\rho_{\mathrm{eq}}=\mathrm{p} \cdot\left(\mathrm{E}[\mathrm{B}]+\frac{\mathrm{D}-1}{2}+\sum_{\mathrm{k}=1}^{\mathrm{D}-1} \frac{1}{\varepsilon_{\mathrm{k}}-1} \frac{\mathrm{pB}\left(\varepsilon_{\mathrm{k}}\right)}{\varepsilon_{\mathrm{k}}-\overline{\mathrm{p}}}\right) .
$$

This quantity can be considered as an equivalent load, in which the effect of the voids, and thus the finite granularity of the FDLs, is already incorporated. (Note that $\rho_{\mathrm{eq}}=1$ when $\mathrm{p}=\mathrm{p}_{\max }$.)

The heuristic derives from the fact that the (steady-state) balance equations for $\operatorname{Pr}[\mathrm{H}=\mathrm{n}]$, in both the finite and the infinite system case, have exactly the same structure for small values of $n$. Up to a scaling factor they will thus also have the same solution. That scaling factor follows easily from the normalization condition for probability distributions. Some further calculations then lead to

$$
\mathrm{LP} \approx \frac{\left(1-\rho_{\mathrm{eq}}\right)}{\rho_{\mathrm{eq}}} \cdot \frac{\operatorname{Pr}\left[\mathrm{H}_{\mathrm{inf}}>\mathrm{N} \cdot \mathrm{D}\right]}{1-\operatorname{Pr}\left[\mathrm{H}_{\mathrm{inf}}>\mathrm{N} \cdot \mathrm{D}\right]} .
$$

The relation only holds approximately, since the equivalence between the balance equations in the finite and infinite case breaks down at the boundary where $\mathrm{n}$ is in the neighborhood of N.D.

Extensive simulations learned, however, that the heuristic (9) is far more accurate than (11) for small loss probabilities, while the opposite holds true for large loss probabilities. (Unfortunately, a mathematical argument that substantiates this observation still has to be found.) Given the former case is of more interest to us here, (9) will be used in the remainder of this paper.

The tail probabilities $\operatorname{Pr}\left[\mathrm{H}_{\text {inf }}>\mathrm{N} \cdot \mathrm{D}\right]$ that appear in (9) can easily be approximated by inversion of the pgf $\mathrm{H}(\mathrm{z})$, by retaining only the dominant poles of that function. It can be shown that

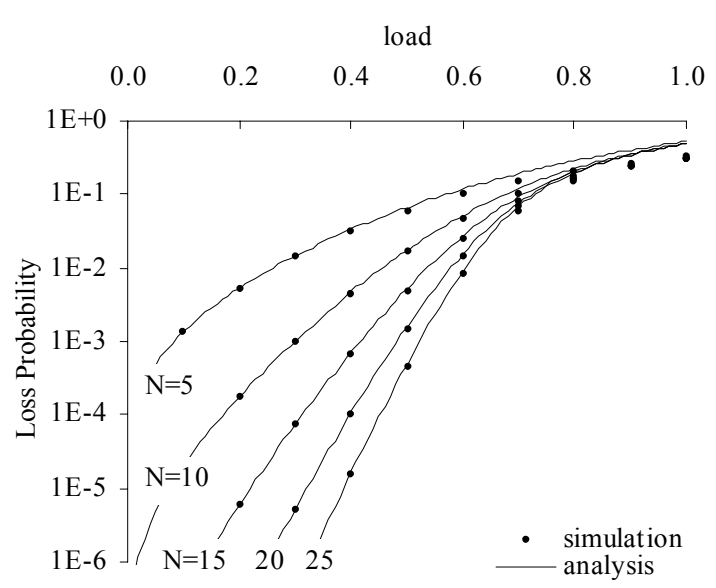

Fig. 2. Loss probability as function of the load, for various FDL buffer sizes N. Burst sizes are geometrically distributed with mean $E[B]=100$ (slots). The FDL granularity $\mathrm{D}=100$ (slots).

$$
\operatorname{Pr}\left[\mathrm{H}_{\mathrm{inf}}>\mathrm{N} \cdot \mathrm{D}\right] \approx \frac{\mathrm{Cst}}{\mathrm{z}_{0} \mathrm{~N} \cdot \mathrm{D}+1},
$$

whereby $\mathrm{z}_{0}$ is the dominant pole along the positive real axis. Its value can easily be determined by means of a simple bisection algorithm. The constant follows from the application of residue theory and is, in its final form, given by

$$
\text { Cst }=\frac{D}{z_{0}{ }^{D}-1} \cdot\left(\lim _{z \rightarrow z_{0}} F(z)\left(z_{0}-z\right)\right) .
$$

The limit in the above can easily be calculated explicitly, its numerical evaluation requires but a few lines of code.

(The function $F(z)$ is related to $H(z)$, see Appendix. Both functions have the same dominant poles. There are $\mathrm{D}$ of them, of the form $\left|z_{0}\right| \cdot \varepsilon_{\mathrm{k}}$ with $\mathrm{k}=0, \ldots \mathrm{D}-1$. The occurrence of multiple dominant poles complicates matters somewhat, as compared to the case of a single one, but only so to a limited extent. For a more detailed explanation, see eg. [9], pp. 147-148, and references therein.)

The accuracy of the above approximation is illustrated in Fig. 2. Shown there is the loss probability LP as function of the load $\rho$, for various FDL buffer sizes. Burst lengths were assumed to be geometrically distributed, with mean $E[B]=100$ (slots), the FDL granularity $\mathrm{D}=100$ (slots). The simulations recorded $10^{8}$ burst arrivals.

Clearly, the accuracy of the approximation is extremely high, even for high loads and small buffer sizes. Note that, for the parameters values used in Fig. 2, the maximum tolerable load $\rho_{\max }$ is about 0.66 . For higher loads, the infinite system becomes unstable, and, as such, the function $\mathrm{H}(\mathrm{z})$ loses its meaning as probability generating function. However, it is possible to "extrapolate" the loss formula for loads above $\rho_{\max }$, 


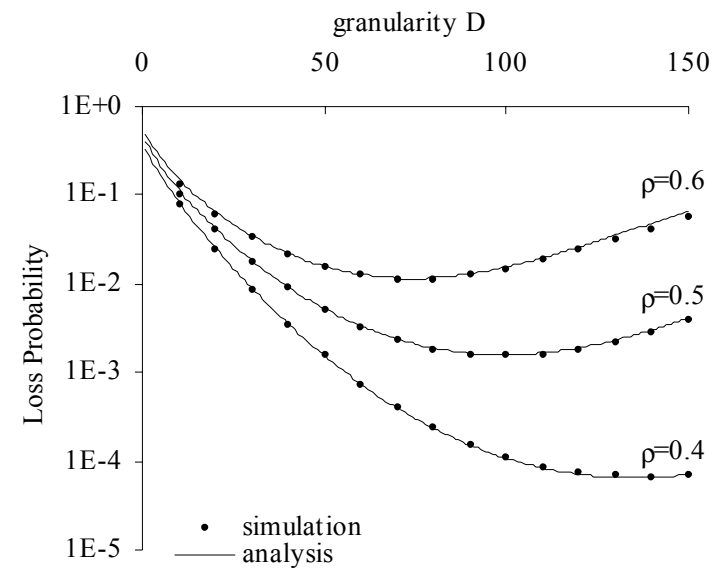

Fig. 3. Loss probability as function of the FDL granularity D (slots), for various load levels, in a buffer of size $\mathrm{N}=20$. Burst sizes are geometrically distributed, with mean $\mathrm{E}[\mathrm{B}]=100$ (slots).

as illustrated in Fig. 2. (For these higher loads, the dominant pole $\mathrm{z}_{0}$ lies within the unit circle, i.e., in the interval $(0,1)$. Further, the constant in the approximation (12) becomes negative, but this is compensated by the fact that $1-\rho_{\mathrm{eq}}$ becomes negative too, so that still a positive loss probability is found.)

\section{Fiber Delay Line Dimensioning}

With the above results at hand, we can now easily study the influence of various design and traffic parameters. We will present two examples, illustrating the sensitivity of the loss performance with respect to the FDL granularity D and with respect to the burst size distribution.

In Fig. 3, the loss probability LP is shown as function of D, for geometrically distributed burst sizes, with mean $\mathrm{E}[\mathrm{B}]=100$ (slots). The size of the buffer was set to $\mathrm{N}=20$. As was already remarked by Callegati [8], there is a trade-off between increasing time resolution of the FDL buffer - smaller values of $\mathrm{D}$ decrease the effect of reduced capacity due to the creation of voids - and maximum achievable delay - which, assuming $\mathrm{N}$ is fixed, can increase only by increasing $\mathrm{D}$. This trade-off leads to an optimal FDL granularity $\mathrm{D}_{\text {opt }}$. The optimum is broad and not very sensitive to the value of $\mathrm{N}$, see also [8]. However, as Fig. 3 clearly illustrates, it is rather sensitive to the offered load. It gradually decreases with increasing load. (For the three load levels shown, the optimum is to be found at $\mathrm{D}_{\mathrm{opt}}=135,99$ and 74 respectively.)

Fig. 4 shows the same curves as Fig. 3, but for deterministic, i.e., fixed burst sizes. The loss probability is about two orders of magnitude smaller than it is for geometrically distributed burst sizes, at a same load level. Again the optimal FDL granularity is function of the load, but now it no longer gradually decreases as the latter increases. For example, when the load increases above 0.59 , the optimum moves from $\mathrm{D}_{\text {opt }}=99$ to $\mathrm{D}_{\text {opt }}=50$. If the load further increases, above $0.77, \mathrm{D}_{\text {opt }}$ becomes 33 , and so on. The same

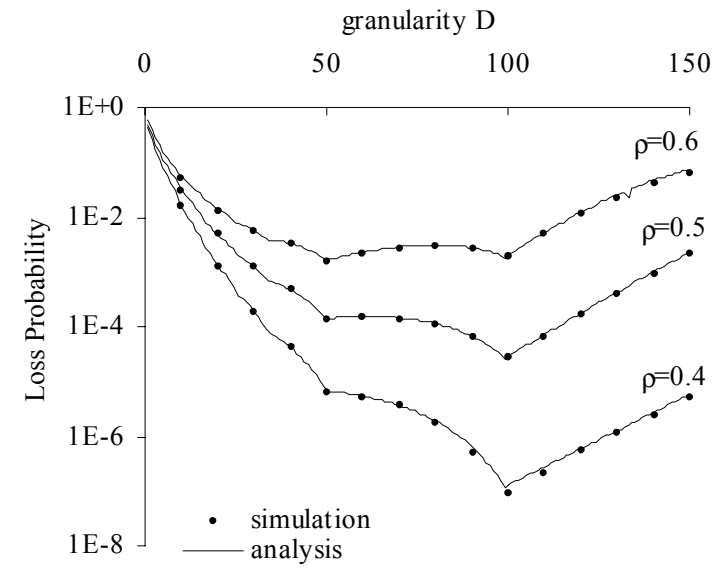

Fig. 4. Loss probability as function of the FDL granularity D (slots), for various load levels, in a buffer of size $\mathrm{N}=20$. Burst sizes are fixed, of length 100 (slots).

behavior occurs (at about the same loads) for different values of the buffer size $\mathrm{N}$, and for different values of the fixed burst size (in which case, however, $\mathrm{D}_{\text {opt }}$ scales accordingly).

\section{CONCLUSION}

The transform-based analysis of the dynamics of a singlechannel optical buffer, as presented above, yields relatively simple formulas for various measures of interest, whose computational burden is close to negligible.

Our study reveals that determining the optimal FDL granularity is a delicate task, whereby one should carefully take into account the parameters of the traffic being offered to the system. A mismatch can seriously degrade the overall performance.

Extensions of the analysis that would allow for more general arrival processes are certainly within reach of the method discussed here, given this extension is, from a mathematical point of view, more or less decoupled from the problems induced by the finite FDL granularity. Similar, but more challenging, is the extension that would allow for multiple channels serving a shared FDL buffer. Evidently, that analysis would run into the same mathematical problems as those of conventional multi-server queues.

\section{APPENDIX}

Deriving $\mathrm{H}(\mathrm{z})$, the equilibrium pgf of the scheduling horizon $\mathrm{H}$ as seen by arrivals - the evolution of which is described by (3) - basically involves two steps.

The first relates to what probably is the most basic problem encountered in queueing theory: given two random variables, say $\mathrm{G}$ and $\tau$, determine the distribution of a third, say $H$, that satisfies the relation

$$
\mathrm{H}=[\mathrm{G}-\tau]^{+}
$$


The complexity of a transform-based solution depends critically on the exact form of the distribution, or equivalently, the pgf of $\tau$, as discussed in e.g. [9]. Here, $\tau$ represents a generic burst interarrival time, and, as mentioned in Section 2, these interarrival times are geometrically distributed here. This leads to the simplest instance of the above problem, and one can easily show that $H(z)$, the pgf of $H$, is related to $G(z)$, that of $\mathrm{G}$, as

$$
H(z)=\frac{p}{z-\bar{p}} G(z)+\frac{z-1}{z-\bar{p}} K .
$$

In the above, $\mathrm{K}$ is a constant that will be determined at the end of our analysis.

The second step in the derivation of $\mathrm{H}(\mathrm{z})$ is related to the finite granularity of the FDLs: given the pgf of $\mathrm{H}$, determine the pgf of $\mathrm{F}$, related to $\mathrm{H}$ through

$$
\mathrm{F}=\mathrm{D} \cdot\left\lceil\frac{\mathrm{H}}{\mathrm{D}}\right\rceil
$$

Using the identity

$$
\sum_{\mathrm{k}=0}^{\mathrm{D}-1} \frac{1}{\mathrm{D}} \varepsilon_{\mathrm{k}}^{\mathrm{m}}= \begin{cases}1 & : \mathrm{m}=\mathrm{i} \cdot \mathrm{D} \text { (i integer) } \\ 0 & : \text { otherwise }\end{cases}
$$

whereby, for notational convenience, we introduced the symbols

$$
\varepsilon_{\mathrm{k}}=\mathrm{e}^{\mathrm{j} 2 \pi \mathrm{k} / \mathrm{D}}
$$

(i.e., the $\mathrm{D}$ different complex $\mathrm{D}^{\text {th }}$ roots of unity) one can show that

$$
\mathrm{F}(\mathrm{z})=\sum_{\mathrm{k}=0}^{\mathrm{D}-1} \frac{1}{\mathrm{D}} \cdot \frac{\left(\mathrm{z} \varepsilon_{\mathrm{k}}\right)^{\mathrm{D}}-1}{\left(\mathrm{z} \varepsilon_{\mathrm{k}}\right)-1} \mathrm{H}\left(\mathrm{z} \varepsilon_{\mathrm{k}}\right)
$$

Basically, identity (18) allows one to selectively map the probabilities $\operatorname{Pr}[\mathrm{H}=\mathrm{n} \cdot \mathrm{D}+\mathrm{m}]$ (for $\mathrm{n} \geq 0$ and $\mathrm{m}=1, \ldots, \mathrm{D}$ ) to the corresponding probabilities $\operatorname{Pr}[\mathrm{F}=(\mathrm{n}+1) \cdot \mathrm{D}]$, as follows from (16). (The case $\operatorname{Pr}[\mathrm{H}=0]$ directly maps to $\operatorname{Pr}[\mathrm{F}=0]$.) In the transform domain, this leads to (19) in a quite straightforward manner. Note also that $\mathrm{F}\left(\mathrm{z} \varepsilon_{\mathrm{k}}\right)=\mathrm{F}(\mathrm{z})$, reflecting the fact that the random variable $\mathrm{F}$ takes on only values that are integer multiples of D.

Combining the above two results, also taking into account the contribution of B in (3), one obtains

$$
H(z)=\frac{p}{z-\bar{p}} B(z) F(z)+\frac{z-1}{z-\bar{p}} K .
$$

Observe that, in light of the remark above,

$$
\begin{aligned}
& \mathrm{H}\left(\mathrm{z} \varepsilon_{\mathrm{k}}\right) \\
& =\frac{\mathrm{p}}{\left(\mathrm{z} \varepsilon_{\mathrm{k}}\right)-\overline{\mathrm{p}}} \mathrm{B}\left(\mathrm{z} \varepsilon_{\mathrm{k}}\right) \mathrm{F}\left(\mathrm{z} \varepsilon_{\mathrm{k}}\right)+\frac{\left(\mathrm{z} \varepsilon_{\mathrm{k}}\right)-1}{\left(\mathrm{z} \varepsilon_{\mathrm{k}}\right)-\overline{\mathrm{p}}} \mathrm{K} \\
& =\frac{\mathrm{p}}{\left(\mathrm{z} \varepsilon_{\mathrm{k}}\right)-\overline{\mathrm{p}}} \mathrm{B}\left(\mathrm{z} \varepsilon_{\mathrm{k}}\right) \mathrm{F}(\mathrm{z})+\frac{\left(\mathrm{z} \varepsilon_{\mathrm{k}}\right)-1}{\left(\mathrm{z} \varepsilon_{\mathrm{k}}\right)-\overline{\mathrm{p}}} \mathrm{K} .
\end{aligned}
$$

Taking the sum over $\mathrm{k}=0 \ldots \mathrm{D}-1$, with appropriate weights, one may thus write

$$
\begin{aligned}
& \mathrm{F}(\mathrm{z}) \\
& =\sum_{\mathrm{k}=0}^{\mathrm{D}-1} \frac{1}{\mathrm{D}} \cdot \frac{\left(\mathrm{z} \varepsilon_{\mathrm{k}}\right)^{\mathrm{D}}-1}{\left(\mathrm{z} \varepsilon_{\mathrm{k}}\right)-1} \mathrm{H}\left(\mathrm{z} \varepsilon_{\mathrm{k}}\right) \\
& =\left(\sum_{\mathrm{k}=0}^{\mathrm{D}-1} \frac{1}{\mathrm{D}} \cdot \frac{\left(\mathrm{z} \varepsilon_{\mathrm{k}}\right)^{\mathrm{D}}-1}{\left(\mathrm{z} \varepsilon_{\mathrm{k}}\right)-1} \frac{\mathrm{pB}\left(\mathrm{z} \varepsilon_{\mathrm{k}}\right)}{\left(\mathrm{z} \varepsilon_{\mathrm{k}}\right)-\overline{\mathrm{p}}}\right) \mathrm{F}(\mathrm{z}) \\
& \quad+\left(\sum_{\mathrm{k}=0}^{\mathrm{D}-1} \frac{1}{\mathrm{D}} \cdot \frac{\left(\mathrm{z} \varepsilon_{\mathrm{k}}\right)^{\mathrm{D}}-1}{\left(\mathrm{z} \varepsilon_{\mathrm{k}}\right)-1} \frac{\left(\mathrm{z} \varepsilon_{\mathrm{k}}\right)-1}{\left(\mathrm{z} \varepsilon_{\mathrm{k}}\right)-\overline{\mathrm{p}}}\right) \mathrm{K},
\end{aligned}
$$

from which $\mathrm{F}(\mathrm{z})$ can then be obtained explicitly. Using the identity

$$
\frac{\mathrm{x}^{\mathrm{D}-1}}{\mathrm{z}^{\mathrm{D}}-\mathrm{x}}=\sum_{\mathrm{k}=0}^{\mathrm{D}-1} \frac{1}{\mathrm{D}} \cdot \frac{1}{\left(\mathrm{z} \varepsilon_{\mathrm{k}}\right)-\mathrm{x}}
$$

to simplify the above expression to some extent, the final result reads

$$
\mathrm{F}(\mathrm{z})=\frac{\mathrm{K} \cdot\left(\frac{\overline{\mathrm{p}}^{\mathrm{D}-1}\left(\mathrm{z}^{\mathrm{D}}-1\right)}{\left.\mathrm{z}^{\mathrm{D}}-\overline{\mathrm{p}}^{\mathrm{D}}\right)}\right.}{\left(1-\sum_{\mathrm{k}=0}^{\mathrm{D}-1} \frac{1}{\mathrm{D}} \cdot \frac{\left(\mathrm{z} \varepsilon_{\mathrm{k}}\right)^{\mathrm{D}}-1}{\left(\mathrm{z} \varepsilon_{\mathrm{k}}\right)-1} \frac{\mathrm{pB}\left(\mathrm{z} \varepsilon_{\mathrm{k}}\right)}{\left(\mathrm{z} \varepsilon_{\mathrm{k}}\right)-\overline{\mathrm{p}}}\right)}
$$

Remains the task of determining the constant $\mathrm{K}$. The function $\mathrm{F}(\mathrm{z})$ is a proper pgf, and is thus normalized, i.e., $F(1)=1$. Assuming $z=1$ in (24) leads to the indeterminate form $0 / 0$, which can, however, be resolved using the rules of de l'Hopital. One finds

$$
\mathrm{K}=\frac{\left(\frac{1}{\mathrm{p}}-\mathrm{B}^{\prime}(1)-\frac{\mathrm{D}-1}{2}-\sum_{\mathrm{k}=1}^{\mathrm{D}-1} \frac{1}{\varepsilon_{\mathrm{k}}-1} \frac{\mathrm{pB}\left(\varepsilon_{\mathrm{k}}\right)}{\varepsilon_{\mathrm{k}}-\overline{\mathrm{p}}}\right)}{\left(\frac{\mathrm{D} \overline{\mathrm{p}}^{\mathrm{D}-1}}{1-\overline{\mathrm{p}}^{\mathrm{D}}}\right)}
$$


Herewith, the pgf $\mathrm{H}(\mathrm{z})$ of $\mathrm{H}$ has been completely determined. As a final remark, note that the constant $\mathrm{K}$ is related to the probability of finding the system empty upon arrival,

$$
\mathrm{H}(0)=\lim _{\mathrm{k} \rightarrow \infty} \operatorname{Pr}\left[\mathrm{H}_{\mathrm{k}}=0\right]=\frac{1}{\overline{\mathrm{p}}} \mathrm{K}
$$

This then allows one to determine the maximum tolerable arrival intensity $\mathrm{p}_{\max }$, as explained in the text.

\section{REFERENCES}

[1] C. Guillemot et al., "Transparent Optical Packet Switching: the European ACTS KEOPS Project Approach", Journal on Lightwave Technology, vol. 16, pp. 2117-2134, Dec. 1998.

[2] J. Turner, "Terabit Burst Switching", Journal on High-Speed Networks, vol. 8, pp. 3-16, 1999.

[3] C. Qiao and M. Yoo, "Optical Burst Switching - A New Paradigm for an Optical Internet", Journal on High-Speed Networks, vol. 8, pp. 69-84, 1999.

[4] Y. Xiong, M. Vandenhoute and H. Cankaya, "Control Architecture in Optical Burst-Switched WDM Networks", IEEE Journal on Selected Areas in Communications, vol. 18, pp 1838-1851, Oct. 2000.

[5] Z. Haas, "The 'Staggering Switch': An Electronically Controlled Optical Packet Switch", Journal on Lightwave Technology, vol. 11, pp. 925-936, May/June 1993.

[6] I. Chlamtac et al., "CORD: Contention Resolution by Delay Lines", IEEE Journal on Selected Areas in Communications, vol. 14, pp. 1014 1029, June 1996.

[7] D. Hunter et al., "SLOB: A Switch with Large Optical Buffers for Packet Switching", Journal on Lightwave Technology, vol. 16, pp. 17251736, Oct. 1998.

[8] F. Callegati, "Optical Buffers for Variable Length Packets", IEEE Communications Letters, vol. 4, pp. 292-294, Sept. 2000.

[9] H. Bruneel and B.G. Kim, Discrete-Time Models for Communication Systems Including ATM., Boston: Kluwer Academic Publishers, 1993. 